\title{
Adiabatic Processes Realized with a Trapped Brownian Particle
}

\author{
Ignacio A. Martínez, ${ }^{1,2}$ Édgar Roldán, ${ }^{1,3,4}$ Luis Dinis, ${ }^{4,5}$ Dmitri Petrov, ${ }^{1,6}$ and Raúl A. Rica ${ }^{1, *}$ \\ ${ }^{1}$ ICFO-Institut de Ciències Fotòniques, Mediterranean Technology Park, 08860 Castelldefels (Barcelona), Spain \\ ${ }^{2}$ Laboratoire de Physique, École Normale Supérieure, CNRS UMR5672 46 Allée d'Italie, 69364 Lyon, France \\ ${ }^{3}$ Max Planck Institute for the Physics of Complex Systems, Nöthnitzerstrasse 38, 01187 Dresden, Germany \\ ${ }^{4}$ GISC-Grupo Interdisciplinar de Sistemas Complejos, Madrid, Spain \\ ${ }^{5}$ Departamento de Física Atómica, Molecular y Nuclear, Universidad Complutense de Madrid, 28040 Madrid, Spain \\ ${ }^{6}$ ICREA-Institució Catalana de Recerca i Estudis Avançats, 08010 Barcelona, Spain
}

(Received 26 September 2014; published 27 March 2015)

\begin{abstract}
The ability to implement adiabatic processes in the mesoscale is of key importance in the study of artificial or biological micro- and nanoengines. Microadiabatic processes have been elusive to experimental implementation due to the difficulty in isolating Brownian particles from their fluctuating environment. Here we report on the experimental realization of a microscopic quasistatic adiabatic process employing a trapped Brownian particle. We circumvent the complete isolation of the Brownian particle by designing a protocol where both characteristic volume and temperature of the system are changed in such a way that the entropy of the system is conserved along the process. We compare the protocols that follow from either the overdamped or underdamped descriptions, demonstrating that the latter is mandatory in order to obtain a vanishing average heat flux to the particle. We provide analytical expressions for the distributions of the fluctuating heat and entropy and verify them experimentally. Our protocols could serve to implement the first microscopic engine that is able to attain the fundamental limit for the efficiency set by Carnot.

Stochastic energetics [1,2] and the fluctuation theorems [3] have been developed as the theoretical framework that studies thermodynamics at small scales, thus establishing the emerging field of stochastic thermodynamics. In parallel, recent advances on micromanipulation and force-sensing techniques [4] have allowed to measure the dynamics and energy changes in physical systems where thermal fluctuations are relevant [5-9] and to test theoretical results derived from stochastic thermodynamics [10-15]. As a major application, miniaturization of thermodynamic engines to single-molecule devices has been possible for the case of Stirling engine [16] or a variety of Maxwell's demons [15,17,18].

Until now, the design of microscopic heat engines has been restricted to those cycles formed by isothermal processes or instantaneous temperature changes [16], where the validity of a heat fluctuation theorem has been tested [19]. Recent works have shown that exerting random forces on a microscopic particle one can accurately tune the effective kinetic temperature of the particle both under equilibrium [20-22] and nonequilibrium driving [23]. However, the application of such a technique to implement nonisothermal processes has not been fully exploited yet [24].

Among all the nonisothermal processes, adiabatic processes are of major importance in thermodynamics since they are the building blocks of the Carnot engine [25]. Microadiabaticity, i.e., true adiabaticity (TA) at the microscopic scale, cannot be realized for single trajectories due to the unavoidable heat flows between microscopic systems and their surroundings. However, a process where no net heat transfer is obtained when averaged over many trajectories, or mean adiabatic (MA) could, in principle, be realized. For simplicity, we will refer in the following MA processes as adiabatic processes.

The notion of microadiabaticity has been studied theoretically since the first models of microscopic heat engines [26]. Schmiedl and Seifert devised a Brownian heat engine with two instantaneous steps in which the positional Shannon entropy of the system is conserved [27]. Further theoretical developments have considered the case of adiabatic processes in the underdamped limit $[28,29]$. The first experimental studies of microscopic heat engines [16] and nonisothermal processes [19] have not realized the case of adiabatic processes in the mesoscale yet.

In this Letter, we report on the realization of quasistatic adiabatic processes with an optically trapped microparticle whose kinetic temperature is controlled by means of an external noisy electric field $[21,24]$. We provide a complete characterization of the thermodynamics of such adiabatic processes. The contributions due to the heat transferred to the momentum degree of freedom are also considered [24], thus adopting the full, underdamped description of the system. Interestingly, we show that doing so does not constitute a trivial extension of the overdamped description, but distinct features clearly arise. In particular, we discuss the shape of the distribution of the fluctuations of heat and entropy, concluding that asymmetries in the heat distributions are a fingerprint of quasistatic nonisothermal processes. 
In $\mathrm{TA}$ processes, the total heat transferred from the environment to the system $Q$ vanishes, and the heat distribution is $\rho_{\mathrm{TA}}(Q)=\delta(Q)$. The fluctuations of the work, $W=\Delta E, E$ being the total energy, are distributed as a piecewise exponential function. In the microscopic regime, one can attain processes where $\langle Q\rangle=0,\langle\cdot\rangle$ denoting average over many realizations in the quasistatic limit. In the latter case, at odds with the TA, the work is delta distributed, $\rho(W)=\delta(\Delta E-\langle Q\rangle)$ [2], whereas the heat is distributed with the same distribution as $\rho_{\mathrm{TA}}(W)$ [30]. Microadiabatic processes are those where the phase space volume is conserved [26]. In the overdamped limit, where changes in the momentum degree of freedom are neglected, such condition is met by keeping the position distribution constant [33]. However, as we discuss below, the overdamped approximation is incomplete when dealing with nonisothermal processes. A process where only the position distribution is conserved is therefore a pseudoadiabatic, since an unavoidable amount of heat is transferred due to the kinetic energy change [27]. In contrast, in the actual adiabatic process, the full phase space volume is conserved and no net heat is transferred to the particle on average [26,28]. Figure 1 illustrates the difference between the evolution of the phase space along both quasistatic pseudoadiabatic and adiabatic processes. Notice that only in the adiabatic process the phase space volume enclosed by the energy surface defined by the system's energy at every moment
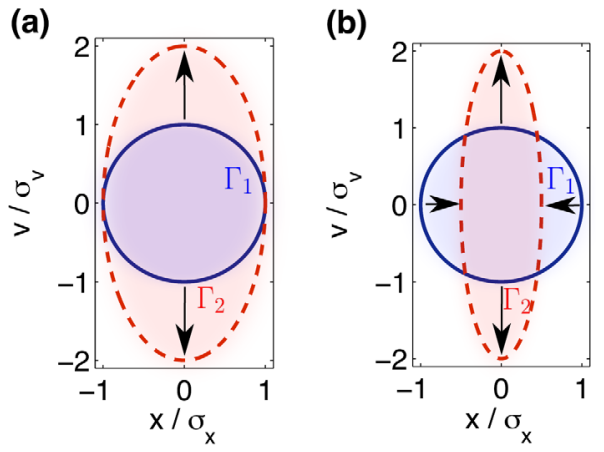

FIG. 1 (color online). Illustration of the pseudoadiabatic and adiabatic processes. A Brownian particle of mass $m$ in a thermal bath at temperature $T$ moves in one dimension $x$ with velocity $v$ and is trapped with a harmonic potential $U(x)=\frac{1}{2} \kappa x^{2}$. The blue solid circle $\Gamma_{1}$ represents the ensemble of microstates described by a Hamiltonian $\mathcal{H}(x, v)=\frac{1}{2} \kappa x^{2}+\frac{1}{2} m v^{2}$ with a given energy $\mathcal{H}(x, v)=E=k T$. Position and velocity are normalized by their standard deviation from the equipartition theorem, $\sigma_{x}=\sqrt{k T / \kappa}$ and $\sigma_{v}=\sqrt{k T / m}$. The red dashed ellipse $\Gamma_{2}$ is the microstate set at the same energy but after two different adiabatic processes: (a) Pseudoadiabatic process, where $T_{\text {fin }}=2 T$ and $\kappa_{\text {fin }}=2 \kappa$ (i.e., $T / \kappa=$ const); (b) adiabatic process, where $T_{\text {fin }}=2 T$ and $\kappa_{\text {fin }}=4 \kappa$ (i.e., $T^{2} / \kappa=$ const). The arrows indicate the direction in which the process occurs.
$\mathcal{H}(x, v)=E$ is conserved, as required for a quasistatic and adiabatic change of parameters [26].

Our system of study is a microparticle of radius $R=500 \mathrm{~nm}$ immersed in water trapped by an optical harmonic optical potential $U(x)=\frac{1}{2} \kappa x^{2}$, where $\kappa$ is the stiffness of the trap and $x$ the position of the particle with respect to the trap center. The key capability of our setup is the independent control of the kinetic temperature of the trapped bead $T_{\text {kin }}$ and $\kappa$, thus allowing one to design a large variety of different thermodynamic processes [23,24]. Both parameters can be electronically synchronized in order to fulfill any desired protocol with high time resolution, of the order of $\mu \mathrm{s}$.

$T_{\text {kin }}$ is defined from the application of equipartition theorem to the fluctuations of the position of the bead in the trap as follows. The mean squared displacement $\left\langle x^{2}\right\rangle$ obeys equipartition theorem, $\kappa\left\langle x^{2}\right\rangle=k T, k$ being Boltzmann's constant and $T$ the temperature of the sample [34]. Applying to the particle an external random force characterized by a Gaussian white noise process of amplitude $\sigma$, we can mimic the kicks of the solvent molecules to the bead in a higher temperature reservoir $T_{\text {kin. }}$. In the presence of such a noise, the kinetic temperature is $T_{\text {kin }}=\kappa\left\langle x^{2}\right\rangle / k=T+\sigma^{2} / 2 \gamma k \geq T, \gamma=6 \pi \eta R$ being the Stokes friction on a sphere in a fluid with kinetic viscosity $\eta$ far away from a surface. These two parameters can be easily controlled, since $\kappa$ is proportional to the intensity of the trapping laser [35] and $T_{\text {kin }}$ increases linearly with the square of the amplitude of a noisy voltage applied to a pair of electrodes in the fluid chamber [30,36]. See Fig. 2(a) for a sketch of the experimental system.

We experimentally realize protocols that conserve either the Shannon entropy in the overdamped approximation, $\mathcal{S}_{x}(t)$ or the full Shannon entropy in the underdamped

\section{(b)}
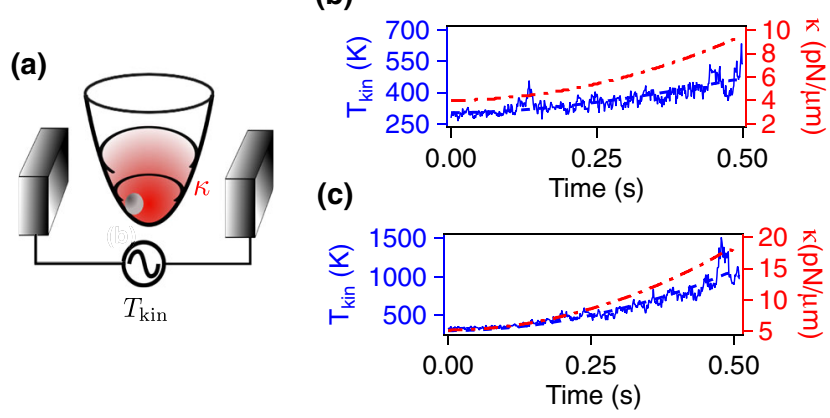

FIG. 2 (color online). Experimental setup and experimental protocols. (a) Sketch of the experimental setup. The kinetic temperature $T_{\text {kin }}$ of a microparticle in an optical trap of stiffness $\kappa$ is controlled with a noisy electric field. (b) Pseudoadiabatic protocol. Kinetic temperature from the mean squared displacement, $\kappa\left\langle x^{2}\right\rangle / k$ (left axis, blue solid line), kinetic temperature from the calibration (left axis, blue dashed line) and stiffness of the trap (right axis, red dash-dot line) as functions of time. (c) The same as (b) but for the adiabatic process. Notice the larger fluctuations as $T_{\text {kin }}$ increases. 
description, $\mathcal{S}(t)$. In the quasistatic limit, the theoretical values for their increments $\Delta \mathcal{S}_{x}(t)=\mathcal{S}_{x}(t)-\mathcal{S}_{x}(0)$ and $\Delta \mathcal{S}(t)=\mathcal{S}(t)-\mathcal{S}(0)$ are, respectively, [30]

$$
\begin{aligned}
\Delta \mathcal{S}_{x}(t) & =\frac{k}{2} \Delta\left(\ln \frac{T_{\text {kin }}(t)}{\kappa(t)}\right), \\
\Delta \mathcal{S}(t) & =k \Delta\left(\ln \frac{T_{\text {kin }}^{2}(t)}{\kappa(t)}\right) .
\end{aligned}
$$

We therefore implement the pseudoadiabatic protocol, where $T_{\text {kin }}(t) / \kappa(t)=$ const [see Fig. 2(b)] and the actual adiabatic protocol, where $T_{\text {kin }}^{2}(t) / \kappa(t)=$ const [see Fig. 2(c)]. All the protocols presented here have a duration of $\tau=0.5 \mathrm{~s}$. Since the relaxation time of the particle in the trap $\tau_{c}$ is of the order of milliseconds [24], then $\tau \gg \tau_{c} \sim \mathrm{ms}$ and the processes can be considered as quasistatic. The latter is confirmed in Figs. 2(b)-2(c), where we show that the measured kinetic temperature fluctuates around the value prescribed by the protocol.

After defining and implementing the desired protocols, we calculate the thermodynamic quantities from measurements of the position of the trapped bead, the stiffness of the trap and the kinetic temperature of the bead, the latter being obtained from standard calibration procedures $[30,36]$. The data acquisition frequency was $f=1 / \Delta t=1 \mathrm{kHz}$. The work done on the particle in the time interval $[t, t+\Delta t]$ is calculated as $\delta W(t)=U\left(x_{t}, t+\Delta t\right)-U\left(x_{t}, t\right), x_{t}$ being the position of the particle at time $t$ [17]. The heat transferred from the thermal bath to the position of the particle is calculated as $\delta Q_{x}(t)=U\left(x_{t+\Delta t}, t+\Delta t\right)-U\left(x_{t}, t+\Delta t\right)$. The internal energy change is measured as the sum of the heat and the work transferred to the particle, $\Delta U(t)=$ $\delta W(t)+\delta Q_{x}(t)=U\left(x_{t+\Delta t}, t+\Delta t\right)-U\left(x_{t}, t\right)$. In the limit $\Delta t \rightarrow 0$, the cumulative sum up to time $t$ of our definitions of heat and work return Sekimoto's expressions $\int \delta W(t) \rightarrow$ $\int(\partial U / \partial t) d t$ and $\int \delta Q_{x}(t) \rightarrow \int(\partial U / \partial x) \circ d x$, where $\circ$ denotes the Stratonovich product [2,30]. Ensemble averages and probability distributions are calculated from data sets of 900 repetitions of each process.

We estimate the kinetic energy changes following the technique described in [24]. The sampling frequency in our experiment is far below the momentum relaxation frequency $f_{p}=\gamma / 2 \pi m \sim \mathrm{MHz}, m$ being the mass of the bead [37]. Therefore, we can only measure time averaged velocities $\bar{v}_{t}=\left(x_{t+\Delta t}-x_{t}\right) / \Delta t$ rather than instantaneous velocities $v_{t}$. In the quasistatic limit, the mean squared instantaneous velocity can be extrapolated from the mean squared time averaged velocity, $\left\langle v_{t}^{2}\right\rangle=\mathcal{L}_{t}\left\langle\bar{v}_{t}^{2}\right\rangle$, where $\mathcal{L}_{t}=\mathcal{L}_{t}\left(f, \kappa_{t}, \gamma, m\right)$ is a function of the sampling frequency as well as of the parameters of the system at time $t$ [24]. The ensemble average kinetic energy change can be therefore calculated as $\left\langle\Delta E_{\text {kin }}(t)\right\rangle=(m / 2)\left[\left\langle v_{t+\Delta t}^{2}\right\rangle-\left\langle v_{t}^{2}\right\rangle\right]=$ $(m / 2)\left[\mathcal{L}_{t+\Delta t}\left\langle\bar{v}_{t+\Delta t}^{2}\right\rangle-\mathcal{L}_{t}\left\langle\bar{v}_{t}^{2}\right\rangle\right]$ [30]. The velocity distributions provide experimental access to the underdamped
Shannon entropy of the system at any time $t,\left\langle S_{t}\right\rangle=$ $-k \int \rho\left(x_{t}, v_{t}, t\right) \ln \rho\left(x_{t}, v_{t}, t\right) d x_{t} d v_{t}[33,38]$, and of the average system entropy change in $[0, t],\langle\Delta S(t)\rangle=\left\langle S_{t}\right\rangle-\left\langle S_{0}\right\rangle$. The overdamped Shannon entropy is obtained neglecting the velocity degree of freedom, $\left\langle S_{x, t}\right\rangle=-k \int \rho\left(x_{t}, t\right) \ln \rho\left(x_{t}, t\right) d x_{t}$.

Using the aforementioned definitions of energetic quantities and entropy, we can now characterize the two types of microadiabatic processes. Figure 3(a) shows ensemble averages of the cumulative sum of work, heat, kinetic energy, internal energy, and total energy for the pseudoadiabatic process, all in agreement with the expected values from equilibrium thermodynamics. The average heat transferred to the position degree of freedom vanishes within experimental errors, yielding a net positive total value of the heat $\langle Q(t)\rangle=\left\langle Q_{x}(t)\right\rangle+\left\langle\Delta E_{\text {kin }}(t)\right\rangle=$ $(k / 2)\left[T_{\text {kin,t }}-T_{\text {kin, } 0}\right]>0$. The average overdamped entropy change vanishes along the protocol, $\left\langle\Delta S_{x}(t)\right\rangle=0$, as shown in Fig. 3(b). The pseudoadiabatic nature of the protocol is revealed as a positive full system entropy change, $\langle\Delta S(t)\rangle>0$ [red curve in Fig. 3(b)]. For the adiabatic protocol [Fig. 2(c)], the ensemble average of the total heat transferred to the particle vanishes within experimental errors, $\langle Q(t)\rangle=0$, as shown in Fig. 3(c). As a result, the system entropy change vanishes along the adiabatic process $\langle\Delta S(t)\rangle=0$ despite entropy is reduced in the position degree of freedom, as shown in Fig. 3(d).
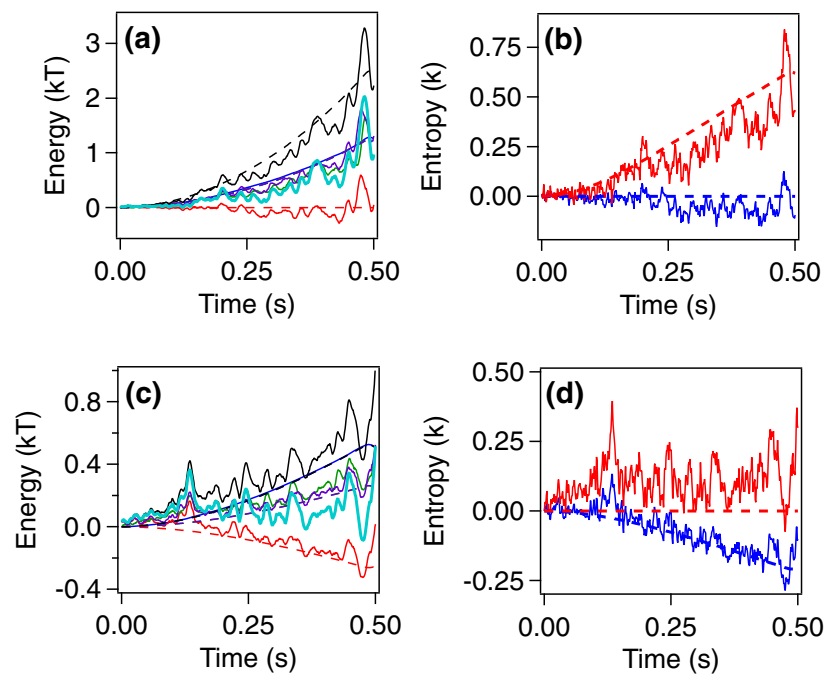

FIG. 3 (color online). Ensemble averages of the cumulative sums of thermodynamic quantities as a function of time in the pseudoadiabatic (a)-(b) and adiabatic (c)-(d) processes. (a) Energy as a function of time for the pseudoadiabatic process, $\langle W(t)\rangle$ (blue), $\left\langle Q_{x}(t)\right\rangle$ (red), $\left\langle\Delta E_{\text {kin }}(t)\right\rangle$ (green), $\langle Q(t)\rangle$ (cyan), $\langle\Delta U(t)\rangle$ (magenta), and $\langle\Delta E(t)\rangle$ (black). (b) System entropy as a function of time for the pseudoadiabatic process, $\left\langle\Delta S_{x}(t)\right\rangle$ (blue) and $\langle\Delta S(t)\rangle$ (red). (c) Energetics of the adiabatic process. (d) System entropy change in the adiabatic process. Dashed curves are the theoretical values of the thermodynamic quantities obtained in the quasistatic limit. 


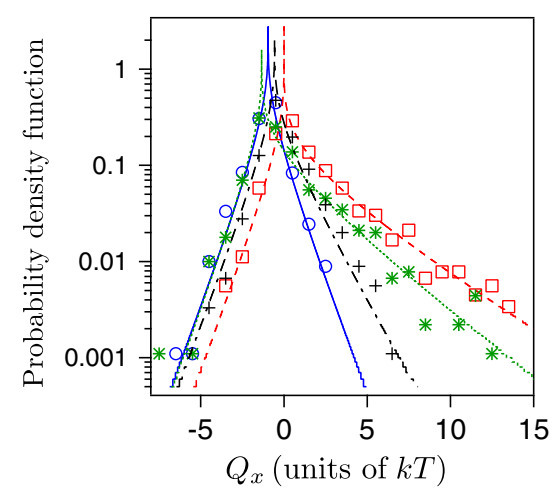

FIG. 4 (color online). Distribution of the heat absorbed by the particle in the position degree of freedom $Q_{x}$ for different thermodynamic processes: isothermal (blue circles), isochoric (red squares), pseudoadiabatic (green stars), and adiabatic (black crosses). The distributions are obtained from 900 cycles. The lines are theoretical distributions obtained from Eq. (3) using the initial and final values of kinetic temperature and stiffness used in the experiments, for the different processes: isothermal (solid line), isochoric (dashed line), pseudoadiabatic (dotted line), and adiabatic (dashed-dotted line).

Let us now consider the fluctuations of the measured quantities. The shape of these distributions reveals qualitative differences between the considered processes. First, in Fig. 4 we show the experimental probability density function of $Q_{x}$ (symbols) for the two adiabatic processes considered. We also include the distributions for two control processes: an isothermal process, where $T_{\text {kin, }}=300 \mathrm{~K}$ while $\kappa$ changes linearly in time from $\kappa_{0}=(5.0 \pm 0.2) \mathrm{pN} / \mu \mathrm{m} \quad$ to $\kappa_{\tau}=(28.0 \pm 0.2) \mathrm{pN} / \mu \mathrm{m}$, and an isochoric process, where $\kappa_{t}=(18.0 \pm 0.2) \mathrm{pN} / \mu \mathrm{m}$ while the kinetic temperature changes linearly from $T_{\text {kin }, 0}=300 \mathrm{~K}$ to $T_{\text {kin }, \tau}=1200 \mathrm{~K}[24]$.

Remarkably, we notice that the heat distribution is asymmetric around its mean for all the nonisothermal processes. The measured heat distributions can be well described by

$$
\rho\left(Q_{x}\right)=\frac{\beta_{G}}{\pi} \exp \left[-\frac{\Delta \beta}{2}\left(Q_{x}+\langle W\rangle\right)\right] K_{0}\left(\bar{\beta}\left|Q_{x}+\langle W\rangle\right|\right),
$$

where $\beta_{0}=1 / k T_{\text {kin }}(0), \beta_{\tau}=1 / k T_{\text {kin }}(\tau), \Delta \beta=\beta_{\tau}-\beta_{0}$, $\bar{\beta}=\left(\beta_{0}+\beta_{\tau}\right) / 2, \beta_{G}=\sqrt{\beta_{0} \beta_{\tau}}, K_{0}$ is the zeroth order modified Bessel function of the second kind and $\langle W\rangle$ is the ensemble average of the work in the quasistatic limit [30]. Equation (3) was obtained with the only assumption of quasistaticity along the process, thus proving that the asymmetry of the distribution of $Q_{x}$ around $-\langle W\rangle$ is a consequence of the nonisothermal character of the process, and not of any nonequilibrium constraint of the system, as suggested in Ref. [19]. For the isothermal case, $\Delta \beta=0$ and we recover the

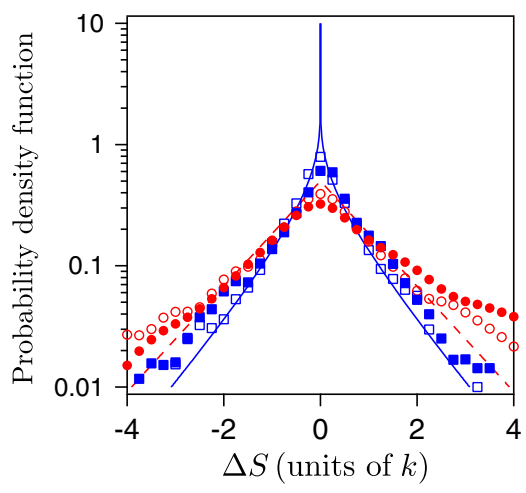

FIG. 5 (color online). Distribution of the system entropy change in the overdamped description, $\Delta S_{x}$ (blue squares) and of the total system entropy change $\Delta S$ (red circles) in the pseudoadiabatic (open symbols) and adiabatic (closed symbols) processes shown in Figs. 2(b) and 2(c). The distributions are obtained from 900 cycles. Theoretical distributions for $\Delta \mathcal{S}_{x}$ [blue solid curve, Eq. (4)] and $\Delta \mathcal{S}$ [red dashed curve, Eq. (5)] are also shown. All the quantities are shifted by their mean such that the mean of the represented quantities is zero.

symmetric distribution $\rho\left(Q_{x}\right)=(\beta / \pi) K_{0}\left[\beta\left|Q_{x}+\langle W\rangle\right|\right]$, first derived by Imparato et al. [39].

The asymmetry observed in the heat fluctuations is not present in the distribution of the entropy. The distribution of the overdamped entropy change along the whole process is symmetric around its mean value for both pseudoadiabatic and adiabatic cases, as shown in Fig. 5. Both distributions fit well to the expected value for general quasistatic nonisothermal processes [30],

$$
\rho\left(\Delta \mathcal{S}_{x}\right)=\frac{1}{\pi k} K_{0}\left(\frac{\left|\Delta \mathcal{S}_{x}-\left\langle\Delta \mathcal{S}_{x}\right\rangle\right|}{k}\right) .
$$

We also calculate the distribution of the full system entropy change along the whole process in both pseudoadiabatic and adiabatic processes (see Fig. 5). System entropy change is distributed symmetrically around its mean value but presents a different qualitative behavior, in this case described by [30]

$$
\rho(\Delta \mathcal{S})=\frac{1}{2 k} \exp \left(-\frac{|\Delta \mathcal{S}-\langle\Delta \mathcal{S}\rangle|}{k}\right) .
$$

Notice that in the case of the full system entropy change, the agreement with the theory extends over one order of magnitude less than in the overdamped description, $\Delta S_{x}$. This mismatch is caused by the poor estimation of the tails of the distribution of the instantaneous velocity from the distribution of the time averaged velocity.

To summarize, we have realized quasistatic adiabatic processes with a single microparticle trapped with optical tweezers. We have studied the difference between the mean pseudoadiabatic (position distribution conserving) and 
mean adiabatic (phase space volume conserving) processes, showing that only the latter are such that the average total heat vanishes in the ensemble average. The fluctuations of the heat transferred to the position of the particle have been shown to be asymmetric for any nonisothermal (equilibrium or nonequilibrium) process. The description of the dynamics of the system with full or limited information affects not only the average values of the entropy but also the fluctuations, showing a different qualitative behavior. The studied microadiabatic protocols could be used to design a microscopic-sized Carnot engine by a cyclic sequence of isothermal and adiabatic processes, thus extending our understanding of micro- and nanoelectromechanical systems towards new and efficient engines $[26,27,40,41]$.

We acknowledge theoretical discussions with J.M. R. Parrondo. I. A. M., E. R., D. P., and R. A. R. acknowledge financial support from the Fundació Privada Cellex Barcelona, Generalitat de Catalunya Grant No. 2009SGR-159, and from grant NANOMQ (MINECO FIS2011-24409). E. R. and L.D. acknowledge financial support from grant ENFASIS (MINECO FIS2011-22644). I. A. M. acknowledges financial support from the European Research Council Grant OUTEFLUCOP. The initial ideas of this work were conceived by Professor D. Petrov, leader of the Optical Tweezers group at ICFO, who has since passed away.

*Corresponding author. rul@ugr.es

[1] K. Sekimoto, Langevin equation and thermodynamics, Prog. Theor. Phys. Suppl. 130, 17 (1998).

[2] K. Sekimoto, Stochastic energetics (Springer, New York, 2010), Vol. 799.

[3] U. Seifert, Stochastic thermodynamics, fluctuation theorems and molecular machines, Rep. Prog. Phys. 75, 126001 (2012).

[4] S. Ciliberto, S. Joubaud, and A. Petrosyan, Fluctuations in out-of-equilibrium systems: from theory to experiment, J. Stat. Mech. 2010, P12003 (2010).

[5] W. Ducker, T. Senden, and R. Pashley, Direct measurement of colloidal forces using an atomic force microscope, Nature (London) 353, 239 (1991).

[6] K. Visscher, M. Schnitzer, and S. Block, Single kinesin molecules studied with a molecular force clamp, Nature (London) 400, 184 (1999).

[7] C. Bustamante, J. Liphardt, and F. Ritort, The nonequilibrium thermodynamics of small systems, Phys. Today 58, 43 (2005).

[8] J. Gieseler, R. Quidant, C. Dellago, and L. Novotny, Dynamic relaxation of a levitated nanoparticle from a non-equilibrium steady state, Nat. Nanotechnol. 9, 358 (2014).

[9] J. Millen, T. Deesuwan, P. Barker, and J. Anders, Nanoscale temperature measurements using non-equilibrium Brownian dynamics of a levitated nanosphere, Nat. Nanotechnol. 9, 425 (2014).

[10] S. Ciliberto and C. Laroche, An experimental test of the Gallavotti-Cohen fluctuation theorem, J. Phys. IV 08, 215 (1998).

[11] J. Liphardt, S. Dumont, S. B. Smith, I. Tinoco Jr., and C. Bustamante, Equilibrium information from nonequilibrium measurements in an experimental test of Jarzynski's equality, Science 296, 1832 (2002).

[12] G. M. Wang, E. M. Sevick, E. Mittag, D. J. Searles, and D. J. Evans, Experimental Demonstration of Violations of the Second Law of Thermodynamics for Small Systems and Short Time Scales, Phys. Rev. Lett. 89, 050601 (2002).

[13] D. Collin, F. Ritort, C. Jarzynski, S. Smith, I. Tinoco, and C. Bustamante, Verification of the Crooks fluctuation theorem and recovery of RNA folding free energies, Nature (London) 437, 231 (2005).

[14] G. M. Wang, J. C. Reid, D. M. Carberry, D. R. M. Williams, E. M. Sevick, and D. J. Evans, Experimental study of the fluctuation theorem in a nonequilibrium steady state, Phys. Rev. E 71, 046142 (2005).

[15] S. Toyabe, T. Sagawa, M. Ueda, E. Muneyuki, and M. Sano, Experimental demonstration of information-to-energy conversion and validation of the generalized Jarzynski equality, Nat. Phys. 6, 988 (2010).

[16] V. Blickle and C. Bechinger, Realization of a micrometresized stochastic heat engine, Nat. Phys. 8, 143 (2011).

[17] É. Roldán, I. A. Martínez, J. M. R. Parrondo, and D. Petrov, Universal features in the energetics of symmetry breaking, Nat. Phys. 10, 457 (2014).

[18] J. Koski, V. Maisi, J. Pekola, and D. Averin, Experimental realization of a Szilard engine with a single electron, Proc. Natl. Acad. Sci. U.S.A. 111, 13786 (2014).

[19] J. R. Gomez-Solano, A. Petrosyan, and S. Ciliberto, Heat Fluctuations in a Nonequilibrium Bath, Phys. Rev. Lett. 106, 200602 (2011).

[20] J. R. Gomez-Solano, L. Bellon, A. Petrosyan, and S. Ciliberto, Steady-state fluctuation relations for systems driven by an external random force, Europhys. Lett. 89, 60003 (2010)

[21] I. A. Martínez, É. Roldán, J. M. R. Parrondo, and D. Petrov, Effective heating to several thousand kelvin of an optically trapped sphere in a liquid, Phys. Rev. E 87, 032159 (2013).

[22] A. Bérut, A. Petrosyan, and S. Ciliberto, Energy flow between two hydrodynamically coupled particles kept at different effective temperatures, Europhys. Lett. 107, 60004 (2014).

[23] P. Mestres, I. A. Martinez, A. Ortiz-Ambriz, R. A. Rica, and E. Roldan, Realization of nonequilibrium thermodynamic processes using external colored noise, Phys. Rev. E 90, 032116 (2014).

[24] É. Roldán, I. A. Martínez, L. Dinis, and R. A. Rica, Measuring kinetic energy changes in the mesoscale with low acquisition rates, Appl. Phys. Lett. 104, 234103 (2014).

[25] S. Carnot, Reflexions on the motive power of fire: A critical edition with the surviving scientific manuscripts (Manchester University Press, Manchester, 1986).

[26] K. Sekimoto, F. Takagi, and T. Hondou, Carnots cycle for small systems: Irreversibility and cost of operations, Phys. Rev. E 62, 7759 (2000). 
[27] T. Schmiedl and U. Seifert, Efficiency at maximum power: An analytically solvable model for stochastic heat engines, Europhys. Lett. 81, 20003 (2008).

[28] S. Bo and A. Celani, Entropic anomaly and maximal efficiency of microscopic heat engines, Phys. Rev. E 87, 050102 (2013).

[29] S. Rana, P. S. Pal, A. Saha, and A. M. Jayannavar, Singleparticle stochastic heat engine, Phys. Rev. E 90, 042146 (2014).

[30] See Supplemental Material at http://link.aps.org/ supplemental/10.1103/PhysRevLett.114.120601, which includes Refs. [31,32], for a description of the experimental setup, protocol to estimate fluctuations of the velocity, and derivations of Eqs. (1)-(5).

[31] K. Visscher, S. P. Gross, and S. M. Block, Construction of multiple-beam optical traps with nanometer-resolution position sensing, IEEE J. Sel. Top. Quantum Electron. 2, 1066 (1996).

[32] P. Langevin, On the theory of Brownian motion, CR Acad. Sci. Paris 146 (1908).

[33] U. Seifert, Entropy Production Along a Stochastic Trajectory and an Integral Fluctuation Theorem, Phys. Rev. Lett. 95, 040602 (2005).
[34] W. Greiner, L. Neise, and H. Stöcker, Thermodynamics and statistical mechanics (Springer, New York, 1999).

[35] A. Mazolli, P. M. Neto, and H. Nussenzveig, Theory of trapping forces in optical tweezers, Proc. R. Soc. A 459, 3021 (2003).

[36] I. Martínez, Noise assisted effects in physics and biophysics studied by the optical trapping technique, Ph.D. thesis, ICFO-Institut de Ciències Fotòniques (2014).

[37] S. Kheifets, A. Simha, K. Melin, T. Li, and M. G. Raizen, Observation of Brownian motion in liquids at short times: Instantaneous velocity and memory loss, Science $\mathbf{3 4 3}, 1493$ (2014).

[38] S. Hilbert, P. Hänggi, and J. Dunkel, Thermodynamic laws in isolated systems, Phys. Rev. E 90, 062116 (2014).

[39] A. Imparato, L. Peliti, G. Pesce, G. Rusciano, and A. Sasso, Work and heat probability distribution of an optically driven Brownian particle: Theory and experiments, Phys. Rev. E 76, 050101 (2007).

[40] N. Sánchez-Salas, L. López-Palacios, S. Velasco, and A. C. Hernández, Optimization criteria, bounds, and efficiencies of heat engines, Phys. Rev. E 82, 051101 (2010).

[41] P. A. Quinto-Su, A microscopic steam engine implemented in an optical tweezer, Nat. Commun. 5, 5889 (2014). 